\title{
Utilização do AutoCAD 2004 para quantificação de pesquisas usando fotomicrografias eletrônicas
}

\section{AutoCAD 2004 to quantify electron-photomicrography}

Carla Albertina Martins Almeida', Silvana Artioli Schellini², Elisa Aparecida Gregório ${ }^{3}$, Cláudia Helena Pellizzon ${ }^{4}$

\section{Resumo}

Objetivo: Avaliar o uso do programa AutoCAD 2004 para estudos quantitativos em microscopia eletrônica. Métodos: A demonstração da utilização do AutoCAD 2004 foi feita em um modelo experimental de diabetes induzido em ratos, divididos em Grupo Controle (GC-10 animais) e Grupo Diabético (GD - 10 animais), avaliados 1 mês depois da indução do diabetes. Os olhos foram removidos após o sacrifício dos animais e preparados para exame em microscópio eletrônico, tendo sido feitas fotografias dos vasos retinianos. Foi utilizado o programa AutoCAD2004 para avaliar a espessura da membrana basal dos vasos. Os resultados foram avaliados estatisticamente. Resultados: Usando o método proposto foi possível realizar medidas quantitativas na membrana basal de vasos retinianos de ratos diabéticos. Conclusão: O AutoCAD 2004 se mostrou efetivo, seguro e de fácil utilização para a quantificação de fenômenos biológicos, sendo possível sugerir este meio para a realização de avaliações quantitativas em experimentos biológicos.

Descritores: Microscopia eletrônica/métodos; Vasos retinianos; Software

\footnotetext{
1Pós-Graduanda da Faculdade de Medicina da Universidade Estadual Paulista "Júlio Mesquita Filho" - UNESP - Botucatu (SP), Brasil; 'Livre-docente do Departamento de Oftalmologia, Otorrinolaringologia e Cirurgia de Cabeça e Pescoço da Faculdade de Medicina da Universidade Estadual Paulista "Júlio Mesquita Filho" - UNESP - Botucatu (SP) - Brasil:

"Professora titular do Departamento de Morfologia do Instituto de Biociências da Universidade Estadual Paulista "Júlio Mesquita Filho" - UNESP - Botucatu (SP), Brasil;

${ }^{4}$ Doutora, Professora assistente do Departamento de Morfologia e Histologia do Instituto de Biociências da Universidade Estadual Paulista "Júlio Mesquita Filho" - UNESP - Botucatu (SP), Brasil.

Trabalho realizado na Faculdade de Medicina da Universidade Estadual Paulista "Júlio Mesquita Filho" - UNESP - Botucatu (SP), Brasil.
} 


\section{INTRODUÇÃO}

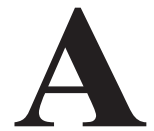

quantificação de elementos estruturais nas fotografias de microscopia eletrônica de transmissão é fundamental nos estudos experimentais e anátomo-patológicos. É freqüente o uso de critérios subjetivos como a classificação de determinadas alterações em "leves, moderadas ou intensas", o que torna difícil a comparação de resultados e a reprodução de experimentos.

Para solucionar falhas como essa, tem sido usada com freqüência crescente a estereologia. A quantificação de elementos estruturais nas fotografias de microscopia eletrônica é fundamental nos estudos experimentais e anátomo-patológicos. A estereologia,também chamada por alguns de morfometria é um conjunto de métodos de quantificação de estruturas morfológicas que permite quantificar numericamente as mais variadas estruturas, macro ou microscópicas ${ }^{(1-5)}$, constituindo-se em instrumento valioso na avaliação de resultados de trabalhos experimentais ${ }^{(6-9)}$.

Vários estudos têm mostrado a utilização de programas de desenho com ajuda do computador, como o AutoCAD (Autodesk, Inc), para realização de medidas diversas em animais e humanos ${ }^{(10-13)}$. Em oftalmologia, alguns trabalhos também têm sido feitos utilizando o AutoCAD como método de medida. ${ }^{(14-15)}$

O objetivo deste é avaliar o uso do programa AutoCAD 2004 para estudos quantitativos em microscopia eletrônica de transmissão.

\section{Métodos}

A demonstração da utilização do recurso quantitativo usando o programa AutoCAD 2004 foi feita em um modelo experimental de diabetes, induzido em 20 ratos albinos, de 3 meses de idade, por administração de Aloxana $2 \%$ (42mg/kg de peso), segundo proto-

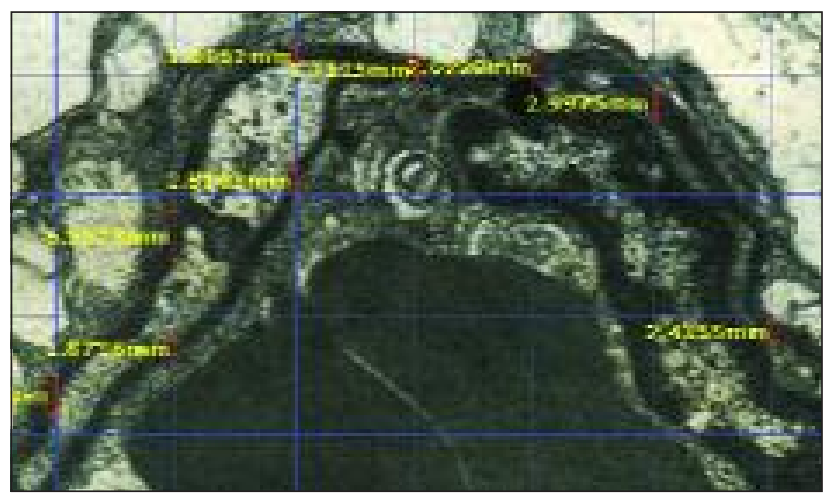

Figura 1: Demonstração do padrão quadriculado de medidas da espessura da membrana basal de vaso retiniano usando o programa)

colo de pesquisa estabelecido na Faculdade de Medicina de Botucatu e que foi aprovado pela Comissão de Ética na Experimentação Animal - UNESP. Os animais foram divididos em Grupo Controle (GC-10 animais) e Grupo Diabético (GD - 10 animais), tendo sido sacrificados 30 dias após a indução da doença. Durante o período experimental, foi realizada avaliação clínico-laboratorial dos animais.

Após o sacrifício dos animais, foi feita a imediata enucleação dos olhos, fixação em glutaraldeído 2,5\%, em tampão fosfato 0,1 M, pH 7,3, pós-fixação em ácido ósmico 1\%, em tampão fosfato 0,1M, pH 7,3, desidratação em série crescente de acetonas (50, 70, 90 e $100 \%$ ) e inclusão dos fragmentos em mistura acetona/Araldite por 12 horas. Foram realizados cortes ultrafinos (500 Angstrons), para exame em microscópio eletrônico de transmissão Philips EM 301. Foram obtidas, ao eletrônico de transmissão, micrografias de vasos retinianos.

As fotografias foram digitalizadas, em local escolhido de forma aleatória, usando o programa AutoCAD 2004, com a utilização de um padrão quadriculado, com medidas padronizadas pelo aumento das fotos (figura 1). Convencionou-se realizar as medidas nos locais de

Tabela 1

Medidas da membrana basal nos grupos controle e diabético

\begin{tabular}{|c|c|c|c|c|c|c|}
\hline \multirow[b]{2}{*}{ Grupo } & \multicolumn{6}{|c|}{$\begin{array}{c}\text { Medida Membrana Basal } \\
\text { (em mm) }\end{array}$} \\
\hline & & Média & & & Variância & \\
\hline $\mathrm{GC}$ & & 2,1009 & & & 2,3089 & \\
\hline GD & & 2,6645 & & & 2,1165 & \\
\hline ANOVA & & & & & & \\
\hline Fonte da variação & SQ & $\mathrm{gl}$ & MQ & F & valor-P & F crítico \\
\hline Entre grupos & 2,16900489 & 1 & 2,169005 & 0,991413 & 0,328238 & 4,210008 \\
\hline
\end{tabular}


cruzamento completo das linhas com a espessura da membrana basal capilar. O número de medidas foi variável de acordo com a quantidade de cruzamentos do quadriculado com a membrana basal. Cada fotografia foi analisada em posição horizontal e vertical para minimizar a variabilidade das medidas devidas à incidência do corte do material.

Os resultados foram avaliados estatisticamente, analisando-se a média e a análise de variância.

\section{Resultados}

As medidas obtidas estão apresentadas na tabela 1. No grupo diabético, os valores apresentaram-se superiores às do grupo controle. Porém, as diferenças não foram estatisticamente significativas.

\section{Discussão}

Os resultados deste estudo sugerem aumento na espessura da membrana basal em animais diabéticos. Entretanto, a tendência de espessamento da membrana basal não foi suficiente para que tivéssemos resultado, estatisticamente significativo. Provavelmente, as alterações que se observa na membrana basal de diabéticos existem com tempo de observação mais prolongado do que o que foi usado no presente estudo ${ }^{(16-19)}$.

A utilização do programa AutoCAD mostrou-se de fácil realização e confiabilidade. Mais importante que os resultados referentes aos animais utilizados para testar o método quantitativo é a constatação de que é possível utilizar este sistema de medida para avaliação quantitativa de variáveis biológicas.

Para que exista confiabilidade no método, as avaliações devem seguir rigorosamente um padrão e serem feitas sempre pelo mesmo observador, além de se considerar as fotomicrografias de forma a não se conhecer a que grupos pertencem os sujeitos da pesquisa.

Desta forma, este método ajudará muito nas futuras análises morfométricas de fotografias de microscopia eletrônica, reduzindo a subjetividade dos exames qualitativos.

\section{CONCLUSÃO}

O programa AutoCAD 2004 se mostrou um método de medida de alta precisão, desde que utilizado com um padrão pré-definido aplicado sobre as imagens, evitando medidas aleatórias, além de ser de fácil utilização para a quantificação de fenômenos biológicos, sendo possível sugerir este meio para a realização de avaliações quantitativas em experimentos biológicos.

\section{Abstract}

Objective: To determine if the AutoCAD 2004 program is available to be used in quantitative research on electron microscopy. Methods: A demonstrative of the AutoCAD2004 was done in an experimental model of diabetes induced in rats. The animals were divided by lot in control group animals (GC-10 animals) and diabetic group (GD - 10 animals), evaluated 1 month after the diabetes induction. The eyes were removed after sacrifice and prepared to electron microscopy. The retina was photographed and the AutoCAD2004 program was used to measure the basal membrane of retina vessels. The results were submitted to statistical analysis. Results: The AutoCAD2004 was effective to obtain quantitative measures from the basal membrane of retina vessels and there were no difference between the studied groups. Conclusion: The AutoCAD2004 was effective, safe and easy to apply to quantify biological parameters, which allowed suggest it to quantitative research.

Keywords: Microscopy, electron/methods; Retinal vessels; Software

\section{REFERÊNCIAS}

1. Elias H, Hyde DM. Guide to pratical stereology. Basel: S Karger; 1983.

2. Gundersen HJ, Bendtsen TF , Korbo L, Marcussen N, Moller A, Nielsen $\mathrm{K}$, et al. Some new, simple and efficient stereological methods and their use in pathological research and diagnosis. APMIS. 1988; 96(5):379-94.

3. Aherne WA, Dunnill MS. Morphometry. London: Arnold; 1982. $205 \mathrm{p}$.

4. Baar JPA, Oort J. A manual of morphometry in diagnostic pathology. Berlin; New York: Springer-Verlag; 1983. p.105-8.

5. Mandarim-de-Lacerda CA. Métodos quantitativos em morfologia. Rio de Janeiro: EDUERJ; 1995. p. 72-81.

6. Baddeley AJ, Gundersen HJ, Cruz-Orive LM. Estimation of surface area from vertical sections. J Microsc. 1986; 142(Pt 3):259-76.

7. Chalkley HW, Cornfield J, Park H. A method for estimating volume-surface ratios. Science. 1949; 110(2856): 295-8.

8. Weibel ER, Gomez DM. A principle for counting tissue structures on random sections. J Appl Physiol. 1962; 17:343-8.

9. Lopes-Paulo F. Emprego da estereologia em pesquisas colorretais. Rev Bras Colo-Proctol. 2002; 22(2):73-6.

10. Biz MT, Figueiredo JA. Morphometric analysis of shank-toflute ratio in rotary nickel-titanium files. Int Endod J. 2004; 37(6):353-8.

11. Masiero AV, Barletta FB. Effectiveness of different techniques for removing gutta-percha during retreatment. Int Endod J. 2005; 38(1):2-7. 
12. Chi FL, Liang Q. The quantification of endolymphatic hydrops in an experimental animal model with guinea pigs. ORL J Otorhinolaryngol Relat Spec. 2004; 66(2):56-61.

14. Yu YS, Ye XD, Shou L. The therapy with rhVEGF gene for ischemic TRAM flap in rats. Zhonghua Zheng Xing Wai Ke Za Zhi. 2003; 19(5):373-6. Id: Chi.

14. Sánchez Pérez A, Honrubia López FM, Larrosa Poves JM, Polo Llorens V, Melcon Sánchez-Frieras B. The Autocad system for planimetric study of the optic disc in glaucoma: technique and reproducibility study. Arch Soc Esp Oftalmol. 2001 76(9):551-8. Id: Spa.

15. Wang N, Lai M, Chen X, Zhou W. Quantitative real time measurement of iris configuration in living human eyes. Zhonghua Yan Ke Za Zhi. 1998; 34(5):369-72. Id: Chi.

16. Weynand B, Jonckheere A, Frans A, Rahier J. Diabetes mellitus induces a thickening of the pulmonary basal lamina. Respiration. 1999; 66(1):14-9.Comment on: Respiration. 1999; 66(1):12-3.

17. Engerman RL, Colquhoun PJ. Epithelial and mesothelial basement membranes in diabetic patients and dogs. Diabetologia. 1982; 23(6):521-4.
18. Silver MD, Huckell VF, Lorber M. Basement membranes of small cardiac vessels in patients with diabetes and myxoedema: preliminary observations. Pathology. 1977; 9(3):213-20.

19. Fisher RF. Factors which influence the thickness of basement membrane in diabetes. Evidence of humoral control. Trans Ophthalmol Soc U K. 1979; 99(1):10-2.

\section{ENDEREÇO PARA CORRESPONDÊNCIA: \\ Silvana Artioli Schellini \\ Faculdade de Medicina de Botucatu \\ Departamento de Oftalmologia \\ Distrito de Rubião Júnior - s/no - Botucatu \\ CEP 18618-970 - São Paulo - SP \\ Tel/Fax: (14)3811 6256}

